\title{
Escala de Atitudes frente à Arma de Fogo (EAFAF): Evidências de Sua Adequação Psicométrica
}

\author{
Thiago Gomes Nascimento \\ Instituto Superior de Ciências Policiais \\ Centro Universitário IESB \\ Carlos Eduardo Pimentel \\ Universidade Federal da Paraíba \\ Breno Geovanni Adaid-Castro \\ Centro Universitário IESB, \\ University of Oregon
}

\begin{abstract}
RESUMO - Este artigo objetivou verificar a validade de construto da Escala de Atitudes Frente à Arma de Fogo (EAFAF). No Estudo 1, participaram 200 policiais militares do Distrito Federal, com idade média de 27,75 anos ( $D P=2,98)$, dos quais 88,8 $\%$ eram homens. Eles responderam a EAFAF e perguntas demográficas. A análise dos componentes principais indicou uma estrutura tri-fatorial, cujos alfas de Cronbach foram 0,81 (direito), 0,76 (proteção) e 0,65 (crime). No Estudo 2, participaram 220 estudantes universitários, com idade média de 24,37 anos $(D P=7,58)$, dos quais $53,0 \%$ eram mulheres. Testou-se a estrutura tri-fatorial por meio de análise fatorial confirmatória. Os indicadores de ajuste foram satisfatórios. Os alfas de Cronbach foram $0,81,0,76,0,75$. O estudo indicou que mulheres tendem a associar armas de fogo a crime, enquanto homens as associam a proteção e direito.
\end{abstract}

Palavras-chave: armas de fogo, atitudes, medidas de atitude, validade do teste

\section{Attitudes Toward Guns Scale (ATGS): Evidence of its Psychometric Adequacy}

\begin{abstract}
This study aimed to verify the construct validity of the Attitude Towards Guns Scale (ATGS). The sample in study 1 was composed by 200 policemen, with a mean age of 27.75 years $(S D=2.98), 88.8 \%$ being men. They responded to the ATGS questionnaire and to demographic questions. A principal components analysis indicated a three-factor structure, with Cronbach's alphas of .81 (justice), .76 (protection) and .65 (crimes). The sample in Study 2 was composed by 220 college students, with a mean age of 24.37 years $(S D=7.58), 53 \%$ being females. The three-factor structure was tested using confirmatory factor analysis. The fit indexes were satisfactory. Cronbach's Alphas of the Study 2 were $.81, .76$ and .75. The study indicates that women tend to associate guns with crime while men tend to associate them with protection and justice.
\end{abstract}

Keywords: firearms, attitudes, attitude measures, validation, test validity

A violência no Brasil tem apresentado, nos últimos anos, um crescimento vertiginoso. Esse fato ocupa cada vez mais espaço nos meios de comunicação, fazendo parte do cotidiano da coletividade, fomentando discussões até mesmo no Congresso Nacional a respeito das armas de fogo, do estatuto do desarmamento, da impunidade, do aumento das penas e da diminuição da idade penal (Peres \& Santos, 2005; Sanches, Duarte, \& Pontes, 2009).

A realidade da violência enfrentada pela população brasileira guarda grande relação com o porte de armas de fogo (Dreyfus \& Nascimento, 2005; Peres, 2004). Estimativas realizadas por Dreyfus e Nascimento (2005) dão conta de que existem, aproximadamente, 17 milhões de armas de fogo nas mãos dos brasileiros, sendo que, desse total, cerca de 5,26 milhões encontram-se nas mãos de criminosos.

Para se ter uma melhor ideia da violência, no período compreendido entre 1999 e 2008, foram registrados 478.369 homicídios no Brasil. Desse total, 332.795 (69,57\%) foram praticados com arma de fogo. A maior média de mortes do período foi registrada no ano de 2003, com 51.043 homicídios registrados no Sistema de Informação sobre Mortalidade

1 Endereço para correspondência: Instituto Superior de Ciências Policiais, Setor Policial Sudoeste, Área Especial 04, Asa Sul, Brasília, DF, Brasil. CEP: 70.610-200.E-mail: nascimento_thiago@outlook.com
(SIM), segundo dados fornecidos pelo Datasus (Ministério da Saúde, 2014).

Uma das formas de se estudar esse problema é por meio das atitudes frente à arma de fogo. No entanto, antes se faz necessário definir as atitudes.

\section{Atitudes}

As atitudes têm sido estudadas há muito tempo na psicologia social e a própria disciplina já foi definida como sendo o estudo científico das atitudes (Ajzen, 2001, Rodrigues, Assmar, \& Jablonsky, 2012). Além disso, as atitudes foram consideradas nas teorias mais importantes da psicologia social, como a teoria do equilíbrio, a teoria da dissonância cognitiva, a teoria do comportamento racional e do comportamento planejado (Rodrigues et al., 2012) ou no modelo MODE (Olson \& Fazio, 2009). Em muitos anos de estudos, uma grande quantidade de objetos foi mensurada e sua utilidade foi destacada para a previsão de comportamentos importantes na sociedade (para uma revisão da importância da mensuração de atitudes veja Pimentel, Torres, \& Günther, 2011). 
Dentre esses objetos psicológicos importantes para o bem-estar e harmonia social, podemos destacar a mensuração de atitudes frente às drogas pesadas (Gouveia, Pimentel, Medeiros, Gouveia, \& Palmeira, 2007), ao álcool (Gouveia, Pimentel, Leite, Albuquerque, \& Costa, 2009), à maconha (Gouveia, Pimentel, Queiroga, Meira, \& Jesus, 2005), à violência (Anderson, Benjamin, Wood, \& Bonacci, 2006), à polícia (Nascimento, Torres, \& Pimentel, 2011), às armas (Branscombe, Weir, \& Crosby, 1991), entre outros construtos. As atitudes podem ser entendidas como avaliações de objetos psicológicos que podem variar de bom a ruim, prejudicial a benéfico ou prazeroso a desprazeroso (Ajzen, 2001; Crites, Fabrigar, \& Petty, 1994) ou como "uma organização duradoura de crenças e cognições em geral, dotada de carga afetiva pró ou contra um objeto social definido, que predispõe a uma ação coerente com as cognições e afetos relativos a este objeto" (Rodrigues et al., 2012, p. 161).

\section{Atitudes Frente à Arma}

As atitudes frente à arma de fogo podem ser importantes para predizer se um indivíduo vai portar armas, para o chamado efeito de armas (Berkowitz \& LePage, 1967; Branscombe et al., 1991), bem como para se entender a violência de forma geral. No entanto, em busca realizada no APA PsycNET (American Psychological Association) por atitudes toward guns and guns no título do documento, encontraram-se apenas sete resultados de interesse, dentre os quais dois trabalhos de pós-graduação e cinco artigos, que incluem a Escala de Atitudes frente à Arma de Fogo de Branscombe et al. (1991), que será tratada no tópico seguinte. Tratou-se, ademais, da predição de atitudes frente às armas de fogo e à violência com base nos estereótipos de gênero; da influência da mídia nas atitudes frente à arma de fogo e no controle de armas; das atitudes dos jovens frente às armas de fogo e à violência, levando-se em consideração o sexo, a idade, o grupo étnico e a exposição à arma de fogo, bem como do desenvolvimento de uma medida para os jovens de atitudes frente à arma de fogo e à violência.

Fazendo a busca por attitudes toward weapons and weapons, nas bases já referenciadas, só apareceram cinco resultados, mostrando a escassez de pesquisas na área. Os estudos envolveram efeitos psicolinguísticos em atitudes frente a armas nucleares; conflito de valores, justificação de valores e atitudes frente a armas nucleares, gênero, papéis de gênero e atitudes frente a guerra e armas nucleares; a relação entre ansiedade frente à morte e atitudes frente a armas nucleares e atitudes frente a armas nucleares e guerra nuclear.

Com o fim de realizar a busca no Brasil, acessou-se o Scielo Brazil e buscou-se, no assunto, dos artigos por atitudes frente à arma, mas não se encontrou qualquer resultado. A mesma busca foi replicada no PePSIC, mas mais uma vez não se encontrou nenhum resultado de interesse.

Esses resultados, em suma, mostram que as atitudes frente às armas foram pouco estudadas. No entanto, alguns estudos se preocuparam em estudar o tema, como se expõe na continuação.

Numa análise sobre as atitudes frente ao controle de arma de fogo, Smith (1980) verificou que $83 \%$ de sua amostra se opuseram ao confisco das armas de fogo e concluiu que as atitudes frente ao controle de armas têm um forte componente de herança cultural, não sendo fortemente alteradas por eventos contemporâneos. Concluiu também que o controle de armas não é visto como uma resposta punitiva ao crime.

Em um primeiro estudo, Shapiro, Dorman, Burkey, Welker, e Clough, (1997) construíram uma medida de atitudes de jovens frente às armas e à violência (Attitudes Toward Guns and Violence Questionnaire - AGVQ). Os autores encontraram quatro fatores principais: Resposta Agressiva à Vergonha, Conforto com Agressão, Entusiasmo e Poder/Segurança, com correlações positivas entre esses fatores variando de 0,28 a 0,54 . $O$ instrumento, que continha inicialmente 61 itens, ficou com 23 após as análises. Os índices de precisão foram considerados satisfatórios, variando de 0,85 a 0,91 . Os jovens que relataram possuir arma apresentaram escores mais altos (1,5 DP) do que aqueles que não possuíam arma (Shapiro et al., 1997).

Em outro estudo, com a utilização do AGVQ, Shapiro, Dorman, Welker, e Clough, (1998) verificaram que os meninos apresentaram mais atitudes frente à arma de fogo e violência comparados às meninas. Além disso, afroamericanos também obtiveram as médias mais altas quando comparados aos caucasianos, estando a exposição à arma de fogo relacionada a mais altas atitudes frente à arma de fogo e violência. As maiores diferenças se deram entre as variáveis sexo, série e exposição à arma e as menores diferenças entre grupo étnico e escola.

Diferentemente dos estudos relatados, Martin et al. (2001) buscaram verificar as associações entre atitudes frente às armas, consumo de álcool e impulsividade. Foi verificado que as atitudes positivas frente às armas se relacionaram com o uso de álcool entre os homens. Verificou-se também que essas atitudes se relacionaram com a impulsividade e o comportamento agressivo, tanto entre os homens como entre as mulheres.

Foram verificados também os efeitos da mídia nas atitudes frente às armas. O autor (Dowler, 2002) identificou que as notícias da TV são a principal fonte para notícias sobre crime. Por meio de regressão logística, o autor verificou que os telespectadores regulares do crime são mais prováveis de apresentarem atitudes negativas ao controle de armas e acreditam que as armas podem prevenir o crime.

No campo da mensuração, sabe-se que pelo menos duas medidas se destinaram a aferir as atitudes frente à arma de fogo: a Escala de Atitudes frente à Arma de Fogo e Violência de Shapiro et al. (1997) e a Escala de Atitudes frente à Arma de Fogo de Branscombe et al. (1991). Embora a medida de Shapiro et al. (1997) tenha apresentado boas qualidades psicométricas, não se trata de uma escala unicamente direcionada a se medir atitudes frente à arma de fogo, mas também à agressão, como acontece com os itens do fator Resposta Agressiva à Vergonha (p.ex.: Se alguém insulta você e você não quer ser um idiota, você tem que lutar). Já a medida de Branscombe et al. (1991), além de apresentar evidências de validade fatorial e precisão, apresenta correlações com várias medidas, falta de correlação com a desejabilidade social e todos os itens se referindo às armas de fogo. Apresenta, portanto, uma estrutura mais parcimoniosa formada por três fatores de atitudes frente às armas de fogo. 
Esta escala, portanto, foi escolhida para ser analisada no presente estudo e é descrita na continuação.

\section{Escala de Atitudes Frente à Arma de Fogo (EAFAF)}

Para Branscombe et al. (1991), a justificativa para criar uma medida de atitudes frente às armas reside no fato de que as crenças e os sentimentos que compõem essas atitudes podem ser considerados importantes moderadores de comportamentos sociais diversos. Para construir a EAFAF, inicialmente Branscombe et al. (1991) formularam 59 itens que deveriam cobrir teoricamente as três dimensões seguintes: (a) Direitos - que envolve afirmações a respeito da posse de armas como um direito fundamental americano (p. ex.: "O direito de portar armas é uma liberdade importante que os americanos deveriam assegurar"); (b) Proteção - que trata de afirmações sobre uma justificativa de se ter armas baseada na proteção com relação a ser vítimas de crimes ( $\mathrm{p}$. ex.: "Os criminosos não atacam as pessoas que possuem armas"); e (c) Crime - que encerra afirmações sobre banir as armas para se evitar o crime (p. ex.: "O acesso fácil a armas de fogo é provável resultar em um aumento da taxa de crime"). Esses itens constituíram um questionário, que utilizou uma escala tipo Likert variando de 1 a 8 pontos (1=baixa discordância a $8=$ alta concordância).

Por meio de uma análise dos componentes principais com rotação varimax, os autores (Branscombe et al., 1991) analisaram inicialmente o conjunto de itens. Verificaram os três fatores conforme descritos acima, com eigenvalues maiores do que 1.9, explicando conjuntamente $60,5 \%$ da variância explicada, foram retidos os itens com cargas fatoriais superiores a 0,50 , retendo-se 17 itens. $\mathrm{O}$ primeiro fator, Direitos, explicou cerca de $36 \%$ da variância explicada. Os autores verificaram também que os três fatores estão relacionados, sendo que Direitos se relacionou moderadamente com Proteção e o fator Crime se relacionou negativamente com esses dois fatores. Posteriormente, os autores inverteram o fator Crime para criar uma pontuação total da escala e relacionaram os três fatores da escala a outras medidas.

Verificaram-se efeitos de gênero nos três fatores e na pontuação total, estando os homens mais convictos de que a arma é um direito do que as mulheres. Eles também acreditam mais na arma como proteção e discordam mais que as armas podem ser a causa do crime, quando comparados às mulheres. Por fim, os homens demonstraram mais atitudes positivas frente às armas do que as mulheres (Branscombe et al., 1991).

Os autores (Branscombe et al., 1991) verificaram correlações positivas dos três fatores da escala com o orgulho americano, percepção como fãs de esportes, falta de confiança nos outros, autoestima e otimismo. Por outro lado, verificaram correlações negativas com orientação política, percepção de que os outros são altruístas, independência de grupos, feminismo, medo, depressão e independência pessoal. Nenhuma das três subescalas nem a pontuação total se correlacionaram com a desejabilidade social, nem com a crença no mundo justo.
Por fim, pode-se verificar que a escala em apreço apresentou coeficientes de precisão satisfatórios, sendo $\alpha=$ 0,89 para o total da escala (com o fator Crime invertido), $\alpha=$ 0,89 para o fator Direitos, $\alpha=0,78$ para o fator Proteção e $\alpha=$ 0,83 para o fator Crime. Verificaram-se ainda uma correlação item-total corrigida variando de 0,54 a 0,70 e correlação da sub-escala (fator) com a escala total variando de 0,69 a 0,87 (Branscombe et al., 1991).

Em suma, parece que existem suficientes evidências de validade e precisão para a EAFAF ser adaptada para estudos no contexto brasileiro. Isso motivou a presente pesquisa como apresentado na continuação. Pretendeu-se testar esta medida em dois estudos independentes para apresentar suas qualidades psicométricas.

\section{Estudo 1. Estrutura Fatorial Exploratória da EAFAF}

Neste primeiro estudo, foram dados os primeiros passos para adaptar a EAFAF (Branscombe et al., 1991) para o contexto brasileiro. Especificamente, procurou-se conhecer evidências de sua validade fatorial e consistência interna, realizando análises exploratórias para checar o número e adequação dos fatores a serem extraídos.

\section{Método}

Participantes. Um total de 200 policiais militares do Distrito Federal fizeram parte desta pesquisa como respondentes. Tais policiais militares foram escolhidos não-aleatoriamente, com predominância do sexo masculino $(87,5 \%)$. Com relação à idade se verificou variação de 20 a 40 anos $(M=27,77 ; D P=2,98)$, sendo $67,6 \%$ da amostra formada por pessoas de 25 a 30 anos de idade. No que diz respeito à escolaridade, observou-se que $75 \%$ possuem graduação e $24 \%$ especialização. A maioria desses policiais são soldados (95\%, primeiro nível hierárquico), solteiros $(64 \%)$ - sendo $24 \%$ casados - , de classe média $(61,8 \%)$ e medianamente religiosa $(42 \%)$.

Instrumentos. Os participantes responderam a um questionário (tipo Likert de 8 pontos, $1=$ discordo totalmente a $8=$ concordo totalmente, conforme estudo original) composto pelas seguintes partes.

- EAFAF: As principais técnicas para tradução de escalas foram consideradas (Cha, Kim, \& Erlen, 2007). Optou-se por tradutores bilíngues. Nesse caso, dois psicólogos brasileiros com vivências nos Estados Unidos e Inglaterra efetuaram traduções independentes. Posteriormente, estas foram comparadas por um terceiro psicólogo bilíngue, tendo em conta a versão original em inglês. Constatando-se concordância entre as traduções, chegou-se à versão aqui apresentada (ver Tabela 2). Aferiu-se sua validade semântica com dez policias militares com mais de 10 anos de atividade policial, que verificaram a se os itens estavam inteligíveis e se havia algum problema para o pleno entendimento do questionário. 
- Questionário sócio-demográfico: Procurou-se conhecer um pouco mais os participantes do estudo, perguntando-lhes essencialmente sua idade, sexo, nível de escolaridade, classe socioeconômica, estado civil, grau de religiosidade e seu nível na hierarquia organizacional.

Procedimento. Os participantes assinaram a um Termo de Consentimento Livre e Esclarecido (TCLE), cujo objetivo era informar aos participantes dos procedimentos éticos para a condução da pesquisa e registrar o seu consentimento.

Após autorização, acerca dos procedimentos éticos do projeto de pesquisa apresentado à Seção de Ciência e Tecnologia do Estado-Maior da Instituição Policial, conforme parecer 003/2013, os instrumentos foram aplicados aos policiais militares em contextos coletivos de sala de aula. As respostas foram dadas individualmente, procurando assegurar-lhes o anonimato e o caráter voluntário da participação. A todos foi dito que, ao preencher e devolver o instrumento, estariam dando o seu consentimento livre e esclarecido para fazer parte do estudo. Aproximadamente cinco minutos foram necessários para concluir sua participação.

Análise dos dados. O PASW (versão 19) foi empregado. Calcularam-se estatísticas descritivas (medidas de tendência central e dispersão) e conduziu-se o teste do poder discriminativo dos itens. Além disso, foi realizada análise de componentes principais (PCA). Nesse ponto, procurou-se previamente avaliar a adequação de se realizar esse tipo de análise, por intermédio de dois critérios: Kaiser-Meyer-Olkin (KMO), que precisa ser de ao menos 0,60 para dar suporte a esse tipo de análise, e o Teste de Esfericidade de Bartlett, cujo valor do qui-quadrado necessita ser estatisticamente significativo (Tabachnick \& Fidell, 2007).

É importante ressaltar que, com o fim de definir o número de fatores a extrair, empregaram-se estratégias mais robustas, isto é, optou-se pela análise paralela (PA) de Horn (1965) e pelo método Hull, desenvolvido por Ceulemans e Kiers (2006) e adaptado para uso na AFE por Lorenzo-Seva, Timmerman e Kiers (2011), em lugar de empregar apenas o critério de Kaiser (valor próprio maior do que 1), que superestima o número de fatores (Hayton, Allen, \& Scarpello, 2004; Laros, 2012). Finalmente, foi calculada a consistência interna (alfa de Cronbach) da estrutura resultante.

\section{Resultados}

Os itens da EAFAF apresentaram médias variando de $1,68(D P=0,95$; Item 13 . Quando você tem uma arma, pode parar de se preocupar em ser vítima de crime) a 6,02 (DP $=1,71$; Item 16. O acesso fácil a armas de fogo é provável resultar em um aumento da taxa de crime). Foi, então, testado o poder discriminativo dos itens.

O critério de mediana empírica para definir os gruposcritério foi empregado. A partir da soma de todos os itens foi possível obter a pontuação total da escala. Disso, definiram-se os grupos inferior e superior, de acordo com as pontuações totais dos participantes, fossem abaixo ou acima da mediana.
Para cada item, foi calculado um teste $t$ comparando as médias dos dois grupos.

Os resultados dos testes $\mathrm{t}$ indicam que todos os itens apresentaram poder discriminativo satisfatório $(p<0,001)$, à exceção de três itens $(12,16$ e 17) que pertencem ao fator crime, conforme a teoria. Diante disso, decidiu-se manter os itens, pelo suporte da teoria, que sustenta o modelo trifatorial (Branscombe et al., 1991). Além disso, Pasquali (2003) observa que, o cálculo do índice de discriminação pelo escore total apresenta um problema teórico, pois busca informações sobre o item baseando-se em todos os itens (escore total), quando ainda não se sabe se os itens do teste são homogêneos. Diante dessas considerações, partiu-se para a verificação de evidências de sua validade fatorial e consistência interna.

Para averiguar a estrutura fatorial da EAFAF avaliou-se a adequação de ser realizada uma análise fatorial, tendo sido os resultados favoráveis: $K M O=0,81$ e Teste de Esfericidade de Bartlett, $\chi^{2}(136)=992,22, p<0,000$. Optou-se então por uma análise de componentes principais (PCA), sem fixar o número de fatores a extrair ou o tipo de rotação. Essa análise permitiu identificar quatro componentes com valores próprios superiores a 1 (critério de Kaiser): 4,92, 1,89, 1,39 e 1,05. A análise do scree plot sugeriu entre três e quatro fatores.

Entretanto, considerando as evidências teóricas e a fragilidade desses critérios, efetuou-se uma análise paralela, considerando uma confiança de $99 \%$ e 1.000 bancos de dados que simularam a presente pesquisa, isto é, 200 participantes e dezessete itens. Contrastando os valores próprios simulados, que resultaram nos seguintes eigenvalues aleatórios: 1,54, $1,43,1,34$ e 1,27, com os valores próprios observados com, percebeu-se que o quarto valor próprio resultado da $\mathrm{CP}(1,05)$ foi inferior àquele simulado $(1,27)$. A partir dos resultados da análise paralela, que tem sido considerada uma técnica mais precisa para a decisão sobre o número de fatores (Hayton et al., 2004; Laros, 2012; Zwick \& Velicer, 1986), indicou-se uma solução tri-fatorial, conforme solução encontrada na versão original do instrumento.

Para corroborar os resultados da PA, procedeu-se a análise de Hull, que tem se apresentado como um método de grande potencial na retenção de fatores (Damásio, 2012). Sua execução envolve a determinação da amplitude de fatores a serem avaliados. Para isso, Lorenzo-Seva et al. (2011) sugerem utilizar o critério das PAs, que indicam a quantidade mínima e máxima de fatores a serem extraídos.

Em seguida, avaliam-se os índices de adequação de ajuste de todas as soluções fatoriais, bem como os graus de liberdade

Tabela 1. Método de Hull para seleção do número de fatores

\begin{tabular}{cccc}
\hline \multicolumn{2}{c}{$\begin{array}{c}\text { Goodness-of-fit index: Método } \\
\text { para extração de dimensões: }\end{array}$} & \multicolumn{2}{c}{$\begin{array}{c}\text { CFI (Comparative Fit Index). } \\
\text { ULS }\end{array}$} \\
\hline $\mathrm{n}^{\text {o de Fatores }}$ & $\begin{array}{c}\text { Valores de } \\
\text { Goodness-of-fit }\end{array}$ & g.l. & $\begin{array}{c}\text { Valores do } \\
\text { Scree test }\end{array}$ \\
0 & 0,000 & 17 & 0,000 \\
1 & 0,685 & 34 & 5,005 \\
2 & 0,814 & 50 & 1,018 \\
3 & 0,932 & 65 & $9.139^{*}$ \\
4 & 0,945 & 79 & 0,000 \\
\hline Nota. * Aconselhamento do número de fatores comuns: 3
\end{tabular}


de cada um dos modelos. As soluções fatoriais são plotadas em um gráfico semelhante ao scree plot, no qual o eixo-y é composto pelos índices de ajuste do modelo, e o eixo-x pelos seus respectivos graus de liberdade. Assim, as soluções que forem representadas fora do limite do fecho convexo (que não sejam os vértices do fecho) são descartadas. Diante disso, um reduzido número de soluções fatoriais tende a ser considerado. A solução fatorial que se encontra no cotovelo (na quebra) do gráfico plotado deve ser retida. Essa mesma solução apresenta o maior valor numérico scree test (st), cuja fórmula matemática pondera a relação entre o índice de adequação de ajuste e os graus de liberdade de um modelo, em comparação com um modelo prévio (ver Ceuleman \& Kiers, 2006; Ceulemans, Timmerman, \& Kiers, 2011; Damásio, 2012; Lorenzo-Seva et al., 2011).

Os resultados apresentados pelo método de Hull indicaram a presença de três fatores (Tabela 1), o que corrobora a estrutura fatorial da escala original, cujos resultados são sumarizados na Tabela 2 .

Como é possível perceber na Tabela 2, a maioria dos itens apresentou saturação superior a 0,30 conforme geralmente adotado na literatura (ver Gorsuch, 1983; Pasquali, 2003). Tal saturação é estatisticamente diferente de zero $(p<0,001)$. Portanto, decidiu-se, nesta oportunidade, assumir o modelo tri-fatorial com dezesseis itens, como proposto na versão original, mesmo com a queda do item 9 (carga fatorial $=0,23$ ), que não atingiu o critério da saturação mínima $(>0,30)$ (Pessoas cometem suicídio frequentemente devido às armas de fogo estarem disponíveis). Essa versão da escala apresentou consistência interna (Alfa de Cronbach) de 0,81 para o Fator 1; de 0,76 para o Fator 2; e de 0,65 para o Fator 3.

O primeiro fator foi formado pelos itens concernentes ao fator Direito, focado em avaliar se a posse de armas deve ser considerado um direito básico do brasileiro; o segundo pelos itens do fator Crime, uma justificação prática para a proibição de armas entre os membros do público em geral, já que as armas estimulam ou causam crimes que não ocorreriam de outra forma; e o terceiro pelos itens do fator Proteção, focado em uma justificação para a posse de armas como uma proteção da vitimização criminal. Juntos os fatores foram responsáveis por explicar $48,21 \%$ da variância explicada. $\mathrm{O}$ primeiro e o segundo fatores apresentaram bons índices de precisão mediante a técnica alfa de Cronbach e lambda 2 de Guttman, entretanto o último fator apresentou índices aceitáveis.

Por fim, foram calculadas as correlações entre os itens dos três fatores da EAFAF. Verificou-se que o primeiro

Tabela 2. Estrutura Fatorial da EAFAF

\begin{tabular}{|c|c|c|c|c|c|}
\hline \multirow{2}{*}{ Itens } & & \multicolumn{3}{|c|}{ Fatores } & \multirow[t]{2}{*}{ h2 } \\
\hline & & 1 & 2 & 3 & \\
\hline 5 & O direito de portar armas é uma liberdade importante que os brasileiros deveriam assegurar. & 0,85 & & & 0,71 \\
\hline 6 & As pessoas deveriam poder ter armas em suas casas. & 0,83 & & & 0,67 \\
\hline 8 & A posse de armas é um valor básico do brasileiro. & 0,51 & & & 0,55 \\
\hline 10 & A proibição de possuir armas representa uma violação à constituição brasileira. & 0,50 & & & 0,44 \\
\hline 11 & As pessoas deveriam ser capazes de possuir armas, porque muitas as usam para fins desportivos. & 0,47 & & & 0,46 \\
\hline 15 & $\begin{array}{l}\text { Independentemente de seu potencial de lesão, é direito de cada pessoa decidir ter ou não uma } \\
\text { arma. }\end{array}$ & 0,47 & & & 0,45 \\
\hline 7 & Eu deveria conseguir facilmente uma arma, se quisesse ter uma. & 0,31 & & & 0,37 \\
\hline 16 & O acesso fácil a armas de fogo é provável resultar em um aumento da taxa de crime. & & 0,75 & & 0,73 \\
\hline 17 & É muito fácil matar quando se tem armas disponíveis. & & 0,72 & & 0,64 \\
\hline 12 & Armas estimulam a criminalidade. & & 0,62 & & 0,61 \\
\hline 1 & Muitos assassinatos não ocorreriam se uma arma não estivesse disponível. & & 0,38 & & 0,44 \\
\hline 9 & Pessoas cometem suicídio frequentemente devido às armas de fogo estarem disponíveis. & & & & 0,71 \\
\hline 13 & Quando você tem uma arma, pode parar de se preocupar em ser vítima de crime. & & & $-0,62$ & 0,54 \\
\hline 3 & A única maneira de garantir que você não será vítima de um crime é possuir uma arma de fogo. & & & $-0,52$ & 0,56 \\
\hline 14 & Possuir uma arma diminui as chances de uma pessoa ser vítima de crime. & & & $-0,49$ & 0,49 \\
\hline 2 & Os criminosos não atacam as pessoas que possuem armas. & & & $-0,45$ & 0,50 \\
\hline \multirow[t]{3}{*}{4} & $\begin{array}{l}\text { Lojistas que têm armas de fogo em suas instalações têm menor probabilidade de serem } \\
\text { assaltados do que aqueles que não têm. }\end{array}$ & & & $-0,38$ & 0,36 \\
\hline & & \multicolumn{3}{|c|}{ Fatores } & \\
\hline & & 1 & 2 & 3 & \\
\hline \multicolumn{2}{|c|}{ Número de Itens } & 7 & 4 & 5 & \\
\hline \multicolumn{2}{|c|}{ Valor Próprio } & 4,92 & 1,39 & 1,89 & \\
\hline \multicolumn{2}{|c|}{ \% de variância explicada } & 28,94 & 8,18 & 11,09 & \\
\hline \multicolumn{2}{|c|}{ Alfa de Cronbach } & 0,81 & 0,65 & 0,76 & \\
\hline \multicolumn{2}{|c|}{ Lambda 2 de Guttman } & 0,82 & 0,66 & 0,76 & \\
\hline
\end{tabular}


fator, direito, apresentou correlação positiva entre os itens variando de 0,55 a 0,81 (rmédia $=0,68$ ); o segundo fator, proteção, apresentou correlação positiva entre os itens de 0,02 a 0,79 (rmédia $=0,57$ ) e o terceiro fator, crime, apresentou correlação positiva entre seus itens de 0,02 a 0,77 (rmédia $=0,57)$.

\section{Estudo 2. Confirmando a Estrutura Fatorial e Consistência Interna da EAFAF}

Como anteriormente sugerido, este estudo procura extrapolar o anterior, contribuindo com procedimentos confirmatórios para checar a adequação da EAFAF. Concretamente, objetivou-se testar a estrutura fatorial dessa medida, verificar a validade convergente e a estabilidade da sua consistência interna (alfa de Cronbach e Confiabilidade Composta).

\section{Método}

Participantes. Um total de 220 estudantes universitários do Distrito Federal fizeram parte desta pesquisa como respondentes. Tais estudantes foram escolhidos nãoaleatoriamente, sendo do sexo feminino 53\%. Com relação à idade, verificou-se variação de 16 a 55 anos $(M=24,53$; $D P=7,67)$, sendo $68 \%$ da amostra formada por pessoas de 19 a 27 anos de idade. No que diz respeito à escolaridade, observou-se que $97,9 \%$ possuem ensino superior incompleto. A maioria desses estudantes são solteiros (83,3\%), de classe média $(52,7 \%)$ e medianamente religiosa $(33,7 \%)$.

Instrumentos. Os participantes responderam a EAFAF, assim como no Estudo 1, mantendo-se a mesma escala de resposta. A manutenção dos 17 itens do estudo exploratório se justifica na medida em que a AFC pode não confirmar a estrutura fatorial obtida com a AFE, entre outros aspectos, pela diferença na extração de fatores, critério de Kaiser e porcentagem da variância explicada, método dos componentes principais $\mathrm{x}$ máxima verossimilhança da AFC (Marôco, 2010). Além disso, responderam as mesmas perguntas demográficas previamente listadas.

Procedimento. Após o preenchimento do TCLE, os instrumentos foram aplicados aos estudantes universitários em contextos coletivos de sala de aula. As respostas foram dadas individualmente, procurando assegurar-lhes o anonimato e o caráter voluntário da participação. A todos foi dito que, ao preencher e devolver o instrumento, estariam dando o seu consentimento livre e esclarecido para fazer parte do estudo. Aproximadamente cinco minutos foram necessários para concluir sua participação.

Análise dos dados. Para comprovação da estrutura fatorial da EAFAF, o AMOS (versão 18) foi utilizado. Considerou-se a matriz de covariâncias como entrada, tendo sido adotado o estimador ML (Maximum Likelihood). Esse tipo de análise estatística é mais rigoroso e criterioso do que o anterior (PCA), possibilitando testar diretamente uma estrutura teórica. No caso, por meio da análise fatorial confirmatória (CFA), testou-se a estrutura fatorial previamente observada (Estudo 1), tomando como referência alguns indicadores de ajuste do modelo teórico com relação aos dados empíricos. Por fim, foi verificado se, para a amostra estudada, existiam diferenças entre gêneros e diferentes idades para as médias dos fatores de proteção, crime e direitos.

Para o teste Qui-quadrado, responsável por averiguar a probabilidade de ajuste do modelo teórico aos dados, foi considerado, em virtude de sua sensibilidade ao tamanho da amostra, a razão em relação aos graus de liberdade $\left(\chi^{2} / g . l\right.$. $)$, cujos valores considerados no intervalo entre 1 e 3 são tidos como satisfatórios. Para o Goodness-of-Fit Index (GFI), que trata da proporção de variância-covariância nos dados, o que o torna semelhante ao $R^{2}$ em regressão múltipla, os valores variam de 0 a 1 , sendo que resultados em torno de 0,90 ou superiores indicam um ajustamento adequado (Byrne, 2001; Marôco, 2010; Tabachnick \& Fidell, 2007).

Foi utilizado para a verificação de um índice comparativo e adicional de ajuste ao modelo, o Comparative Fit Index (CFI), em que valores mais próximos de 1 indicam melhor ajuste; recomendando-se valores de 0,90 ou superiores. Fezse uso, ainda, do Root-Mean-Square Error of Approximation (RMSEA), com seu intervalo de confiança de 90\% (IC90\%); analisado para valores inferiores a 0,08 , mesmo aceitandose valores próximos de 0,10 (Byrne, 2001; Marôco, 2010; Tabachnick \& Fidell, 2007).

Por fim, foi analisado o Standardized Root Mean Square Residual (SRMR), como uma medida absoluta de ajuste, definida como a diferença normalizada entre a correlação observada e à correlação previsível. Um valor inferior a 0,08 é considerado geralmente um bom ajuste (Hu \& Bentler, 1999).

Foi ainda calculada a variância média extraída (VME), que fornece evidências complementares de validade de construto (Fornell \& Larcker, 1981; Hair, Anderson, Tatham, \& Black, 2005, Marôco, 2010, Peterson \& Kim, 2013). Ademais a confiabilidade composta (CC), segundo Peterson e Kim (2013), apresenta índices de confiabilidade, em média, maiores do que aqueles produzidos pelo coeficiente alfa, porque o coeficiente alfa apresenta-se como uma versão restrita da confiabilidade composta. Valores iguais ou superiores a 0,50 para a VME e 0,70 para a CC, respectivamente, garantem a adequação das medidas. A VME é considerada uma medida da validade convergente do fator, ou seja, o quanto ele serve para explicar o conjunto de itens; a CC pode dirimir dúvidas quanto ao alfa de Cronbach, que é influenciado pelo número de itens (Pasquali, 2003).

\section{Resultados}

Procurou-se testar o modelo tri-fatorial previamente encontrado no Estudo 1. Nesse sentido, realizou-se uma análise fatorial confirmatória admitindo que o conjunto dos itens saturam em três fatores correlacionados. Os indicadores de ajuste do modelo, apesar de não terem sido extraordinários, foram bastante promissores: $\chi^{2}(116)=237,08, p<0,000$; $\chi^{2} /$ g.l. $=2,04 ; G F I=0,88 ; C F I=0,91 ; R M S E A=0,069$ $(I C 90 \%=0,56-0,82)$ e $S R M R=0,062$.

Ao se analisar a matriz dos resíduos estandardizados, o item 9 (Pessoas cometem suicídio frequentemente devido às armas de fogo estarem disponíveis) apresentou o valor superior ao limite de $|2,58|$, o que pode indicar problemas 


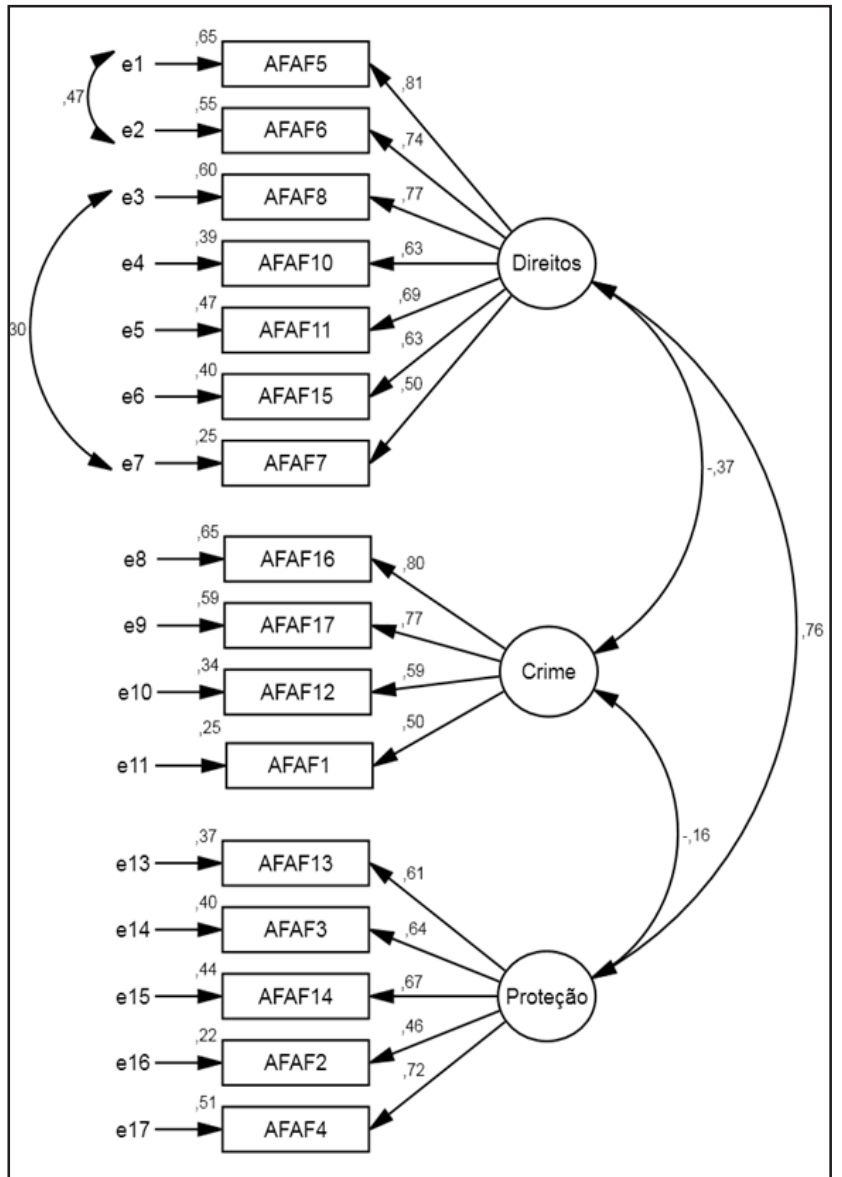

Figura1. Estrutura Fatorial da Escala de Atitudes Frente à Arma de Fogo (EAFAF)

no ajustamento. A correção pode-se dar pela eliminação do indicador em questão (MacCallum, 1986), como aconteceu com o Estudo 1. Esse resultado confirma que o item não se aplica às amostras estudadas.

Não obstante, decidiu-se reespecificar o modelo sem o item 9, considerando os IMs (Índices de Modificação). Concretamente, reespecificaram-se as covariâncias entre dois pares de erros de medida do primeiro fator. Com essas modificações, obtiveram-se melhores índices de ajuste: $\chi^{2}$ $(99)=142,43, p=0,003 ; \chi 2 /$ g.l. $=1,44 ; G F I=0,93 ; C F I$ $=0,97 ; R M S E A=0,045(I C 90 \%=0,027-0,060)$ e $S R M R=$ 0,054 . A estrutura fatorial confirmatória final correspondente pode ser vista na Figura 1.

Todos os itens da EAFAF apresentaram saturações (pesos fatoriais, $\lambda)$ estatisticamente diferentes de zero $(\lambda \neq 0 ; z>1,96$, $\mathrm{p}<0,05)$. Portanto, parecem existir evidências de validade fatorial dessa medida. Sua validade convergente também ficou evidenciada, sendo os valores da $V M E=0,50$ para os Fatores 1 (Direitos), 2 (Crime) e 3 (Proteção).

Conhecida a estrutura fatorial da medida de atitudes frente à arma de fogo, faz-se necessário obter informações sobre sua precisão, avaliada por meio de dois indicadores: consistência interna (alfa de Cronbach, $\alpha$ ) e homogeneidade (correlação média item-total corrigida, ri.t). Enquanto o primeiro avalia a totalidade do fator, apresentando sensibilidade ao número de itens, o segundo considera item a item, possibilitando que se julguem o conjunto de itens e cada item separadamente.
Os resultados dessas análises foram os seguintes: Fator 1 (Direito): $\alpha=0,87$ e o coeficiente médio ri.t $=0,65$, variando de 0,49 (item 7) a 0,76 (item 5). A confiabilidade composta foi de 0,87 . Para o Fator 2 (Crime), $\alpha=0,75$ e o coeficiente médio ri.t $=0,53$, variando de 0,45 (item 1) a 0,64 (item 16). A confiabilidade composta foi de 0,80 . Finalmente, para o Fator 3 (Proteção), $\alpha=0,76$ e o coeficiente médio ri.t $=0,53$, variando de 0,42 (item 2) a 0,61 (item 4). A confiabilidade composta foi de 0,83 . Todos os índices aqui relatados corroboraram os indicadores de confiabilidade anteriormente citados.

Considerando a amostra de estudantes $(n=220)$, foi realizada uma MANCOVA com os fatores da EAFAF como variáveis dependentes, o sexo como variável antecedente e a idade como covariável. Constatou-se um efeito multivariado do sexo do participante $[\lambda$ de Wilks $=0,89, F(3,196)=8,085$, $p=0,000, \eta 2$ parcial $=0,11$, poder $=0,99]$ sobre cada um das dimensões do construto.

Observou-se que os homens apresentaram maior média para os fatores proteção $[M=3,11, D P=1,18 ; F=10,44$, $p=0,001, \eta 2$ parcial $=0,05$, poder $=0,90]$ e direito $[M=3,52$, $D P=1,69 ; F=20,18, p=0,000, \eta 2$ parcial $=0,092$, poder $=0,99]$, enquanto as mulheres apresentaram a média mais elevada para o fator crime $[M=5,42, D P=1,28 ; F=12,92, p=0,000, \eta 2$ parcial $=0,061$, poder $=0,95]$. Para os três fatores estudados, a idade não apresentou efeito significativo.

\section{Discussão}

O presente artigo procurou reunir evidências de validade de construto (validade fatorial, alfa de Cronbach, homogeneidade e confiabilidade composta) da EAFAF (Branscombe et al., 1991). Os dois estudos realizados utilizam um instrumento simples e autoaplicável, que necessita somente de lápis e papel, cujos itens isoladamente ou no conjunto apresentam qualidades métricas satisfatórias, o que sugere seu emprego em pesquisas futuras.

Conforme destacado previamente, as atitudes frente às armas de fogo são um construto bastante relevante para a compreensão do motivo pelos quais uma pessoa, às vezes, mostra um aumento da agressividade na presença de uma arma e, às vezes, mostra uma agressão reduzida (Branscombe et al., 1991). Nesse sentido, confia-se oferecer uma contribuição importante à temática, favorecendo que se avaliem objetivamente as atitudes frente às armas, contribuindo-se para a área da mensuração de atitudes no contexto nacional (Pimentel et al., 2011). Embora as amostras de conveniência demandem a replicação desses estudos em outras cidades brasileiras, a congruência dos resultados sugere que os achados merecem atenção. Portanto, procurase, a seguir, discuti-los.

Os achados previamente descritos sugerem que a versão brasileira, composta por dezesseis itens, é parcimoniosa, descrevendo três fatores que corroboram a estrutura encontrada no estudo original (Branscombe et al., 1991). Destaca-se que tal estrutura emergiu na análise fatorial exploratória (PCA) e foi suportada com a análise fatorial confirmatória (ML), cujos indicadores de ajustamento estão dentro dos valores recomendados na literatura (Byrne, 2001; 
Garson, 2003; Marôco, 2010). Esse instrumento também apresentou evidências de validade convergente, como avaliada por intermédio da VME (Fornell \& Larcker, 1981; Hair et al., 2005; Marôco, 2010).

No que se refere aos indicadores de consistência interna, os alfa de Cronbach observados foram superiores ao valor recomendado na literatura (0,70; Pasquali, 2003). Entretanto, ao se analisarem os índices de consistência interna, nos dois estudos, é possível verificar que, quando considerada a amostra de policiais, o valor de $\alpha(0,65)$ para o fator "Crime" foi menor do que o recomendado na literatura.

A análise do conteúdo do Fator Crime (justificação prática para a proibição de armas entre os membros do público em geral, já que as armas estimulam ou causam crimes que não ocorreriam de outra forma) e dos itens que o compõem, possibilita uma interpretação diferente para os dois grupos pesquisados. No caso dos policiais, a percepção sobre o acesso, a posse e o estimulo à criminalidade quando da presença da arma de fogo é um dos aspectos que influenciam a prática do crime, mas não o único. Essa constatação encontra respaldo no próprio SIM, fornecido pelo Datasus/Ministério da Saúde, em que mortes decorridas de armas brancas (facas), por brigas e por acidentes de trânsito, contribuem para que os policiais apresentem parâmetros atitudinais diversos, por exemplo, ao se comparar com estudantes (Estudo 2), cuja amostra apresentou índices de consistência interna superior $(\alpha=0,75)$. A interpretação desse resultado pode residir no fato de os estudantes atribuírem maior peso, para a realização do crime, à presença da arma de fogo.

A consistência interna do Estudo 2 foi similar ao encontrado por Branscombe et al. (1991) em outro contexto cultural, justificando a utilização dessa medida no contexto de pesquisa, quando o propósito for conhecer atitudes frente às armas de fogo. Ademais, observaram-se que os indicadores de homogeneidade são coerentes com o que sugere a literatura (Clark \& Watson, 1995), sendo a confiabilidade composta situada na faixa superior ao limite de 0,70 estabelecido pela literatura (Hair et al., 2005), tratando-se de uma avaliação complementar de evidências empíricas desse parâmetro psicométrico.

No que se refere à reespecificação do modelo da Figura 1, pelos IMs, a justificativa para sua modificação está na proximidade teórica dos itens, cujos erros foram correlacionados (item 5 e item 6; item 7 e item 8), essa medida é possível, na medida em que, os itens refletem o mesmo fator (Direitos). Ao apresentarem-se no mesmo fator, os termos de erros correlacionados somente alteram a distribuição dos pesos fatoriais, não alterando a estrutura do construto (Marôco, 2011), possibilitando sua alteração sem interferir na medida.

Conforme encontrado nos estudos anteriores de Branscombe et al. (1991) e Shapiro et al. (1998), homens e mulheres diferem em suas atitudes frente à arma de fogo. As mulheres percebem as armas como susceptíveis a estimular o crime e se mostram menos propensas a acreditar na proteção ao se portar uma arma ou apoiar o porte como um direito, como foi encontrado para os homens da amostra.

Pesquisas futuras são recomendadas, embora a estrutura da EAFAF apresente evidências psicométricas favoráveis. Nesse sentido, poderia ser pensada a possibilidade de reescrever o item que foi eliminado no Estudo 1, mas que se manteve no Estudo 2 (item 9), ou ainda elaborar novos itens que cubram toda a extensão do construto atitudes frente à arma de fogo. Outra lacuna que merece atenção e que possibilitará um avanço para estabelecer a adequação dessa medida é conhecer sua validade de critério (concorrente e, principalmente, preditiva). Ademais, pode-se checar a validade convergente e discriminante desse instrumento e avaliar sua estabilidade temporal (teste-reteste), o que pode ser bastante oportuno (Pasquali, 2003), analisando a magnitude, sua flutuação com o tempo ou eventos históricos identificados (Fonsêca et al., 2013).

Finalmente, sugere-se o uso da EAFAF no âmbito educacional atrelado a medidas que enfatizem aspectos quotidianos de adolescentes, jovens e adultos, como atitudes frente à violência (Anderson et al., 2006), consumo de álcool (Martin et al., 2001), preferência musical (Pimentel, 2004), condutas antissociais e delitivas (Santos, 2008), atitudes frente à polícia (Nascimento et al., 2011) ou mesmo frente a medidas que visam aferir a qualidade do serviço de segurança pública (Faiad, Delabrida, \& Nascimento, 2011). Não obstante, não se descarta o emprego da EAFAF como medida de screening (triagem), que, conjuntamente com outras, permitiria identificar grupos de risco potenciais, quer no âmbito educacional ou de segurança pública.

Por fim, devem ser implementados também estudos correlacionando essa medida de atitudes frente às armas de fogo com uma medida de atitudes implícitas (Olson \& Fazio, 2009) frente a esse objeto social. Esses e outros esforços podem ser verdadeiramente úteis para melhor se compreender a mensuração das atitudes frente às armas de fogo.

\section{Referências}

Ajzen, I. (2001). Nature and operation of attitudes. Annual Review of Psychology, 52, 27-58.

Anderson, C. A., Benjamin, A. J., Wood, P. K., \& Bonacci, A.M. (2006). Development and testing of the Velicer Attitudes Toward Violence Scale: Evidence for a four-factor model. Aggressive Behavior, 32, 122-136.

Byrne, B. M. (2001). Structural equation modeling with AMOS: Basic concepts, applications, and programming. Mahwah: Lawrence Erlbaum Associates.

Berkowitz, L., \& LePage A (1967). Weapons as aggression-eliciting stimuli. Journal of Personality and Social Psychology, 7, 202-207.

Branscombe, N. R., Weir, J. A., \& Crosby, P. (1991), A three-factor scale of attitudes toward guns. Aggressive Behavior, 17, 261-273. doi: 10.1002/1098-2337.

Brasil, Ministério da Saúde. Banco de dados do Sistema Único de Saúde-DATASUS. Recuperado de http://www.datasus.gov.br

Ceulemans, E., \& Kiers, H. A. L. (2006). Selecting among threemode principal component models of different types and complexities: A numerical convex hull based method. British Journal of Mathematical \& Statistical Psychology, 59, 133150. doi: 10.1348/00711005X64817. 
Ceulemans, E., Timmerman, M. E., \& Kiers, H. A. L. (2011). The CHull procedure for selecting among multilevel component solutions. Chemometrics and Intelligent Laboratory Systems, 106, 12-20. doi: 10.1016/j.chemolab.2010.08.001.

Cha, E. S., Kim, K. H., \& Erlen, J. A. (2007). Translation of scales in cross-cultural research: issues and techniques. Journal of Advanced Nursing, 58(4), 386-395.

Clark, L. A., \& Watson, D. B. (1995) Constructing validity: Basic issues in objective scale development. Psychological Assessment, 7, 309-319.

Crites S. L., Fabrigar, L. R., \& Petty, R. E. (1994). Measuring the affective and cognitive properties of attitudes: Conceptual and methodological issues. Personality and Social Psychology Bulletin, 20(6), 619-34.

Damasio, B. F. (2012). Uso da análise fatorial exploratória em psicologia. Avaliação Psicológica, 11(2), 213-228.

Dowler, K. (2002). Media influence on attitudes toward guns and gun control. American Journal of Criminal Justice, 26(2), 235-247.

Dreyfus, P, \& Nascimento, M. S. (2005). Small Arms Holdings in Brazil: Toward a Comprehensive Mapping of Guns and Their Owners. In R. Fernandes (Ed.), Brazil: The Arms and the Victims (pp. 94-145). Rio de Janeiro: 7 Letras/Viva Rio/ISER.

Faiad, C., Delabrida, Z. N., \& Nascimento, T. G. (2011). Survey sobre a avaliação da qualidade do serviço de segurança oferecido pela Polícia Militar segundo proprietários e funcionários de comércio em Brasília. Psicologia em Pesquisa, 5(1), 77-85.

Fornell, C., Larcker, D. F. (1981). Evaluating structural equation models with unobservable variables and measurement error. Journal of Marketing Research, 18(1), 39-50.

Garson, G. D. (2003). PA 765 Statnotes: An online textbook. Recuperado de http://www2.chass.ncsu.edu/garson/pa765/ statnote.htm.

Gorsuch, R. L. (1983). Factor analysis (2nd ed.). Hillsdale, NJ: Erlbaum.

Gouveia, V. V., Pimentel, C. E., Leite, P. R. L., Albuquerque, J. R., \& Costa, T. A. B. (2009). Escala de atitudes frente ao uso de álcool: Evidências de validade fatorial e preditiva. Psicologia: Ciência \& Profissão, 29(4), 672-685.I

Gouveia, V. V., Pimentel, C. E., Medeiros, E. D., Gouveia, R. S., \& Palmeira, J. N. (2007). Escala de atitudes frente ao uso de drogas: Evidências de validade fatorial e preditiva. Jornal Brasileiro de Psiquiatria, 56(1), 53-59.

Gouveia, V. V., Pimentel, C. E., Queiroga, F., Meira, M., \& Jesus, G. R. (2005). Escala de atitudes frente ao uso de maconha: Comprovação da sua validade de construto. Jornal Brasileiro de Psiquiatria, 54(1), 5-12.

Hair, J. F., Anderson, R. E., Tatham, R. L., \& Black, W. C. (2005). Análise multivariada de dados (A. S. Sant'Anna \& A. C. Neto, trad.). Porto Alegre: Bookman.

Hayton, J. C., Allen, D. G., \& Scarpello, V. (2004). Factor retention decisions in exploratory factor analysis: A tutorial on parallel analysis. Organizational Research Methods, 7(2), 191-207. doi: $10.1177 / 1094428104263675$.

Horn, J. (1965). A rationale and test for the number of factors in factor analysis. Psychometrika, 30(2), 179-185.

Hu, L. \& Bentler, P. M. (1999). Cutoff criteria for fit indexes in covariance structure analysis: Coventional criteria versus new alternatives. Structural Equation Modeling, 6(1), 1-55.
Laros, J. A. (2012). O uso da Análise Fatorial: Algumas diretrizes para pesquisadores. In L. Pasquali (Ed.), Análise Fatorial para pesquisadores (1ed, pp 141-160). Brasília: LabPAM Editora.

Lorenzo-Seva, U., Timmerman, M. E., \& Kiers, H. A. (2011). The hull method for selecting the number of common factors. Multivariate Behavioral Research, 46(2), 340-364. doi:10.1080/00273171.

MacCallum, R. C. (1986). Specification searches in covariance structure modeling. Psychological Bulletin, 100(1), 107-120. doi. 10.1037/0033-2909.100.1.107.

Marôco, J. (2010). Análise de equações estruturais: Fundamentos teóricos, software e aplicações. Perô Pinheiro: Report Number.

Martin, C. A., Mainous, A. G., Ford, H. H., Mainous, R., Slade, S., Martin, D., \& Omar, H. (2001). Attitudes toward guns: Associations with alcohol use and impulsive behaviors. International Journal of Adolescent Medicine and Health, 13(3), 205-210.

Ministério da Saúde. Datasus. (2014). Informações de Saúde. Mortalidade. Disponível na Internet: http://www2.datasus. gov.br/DATASUS/index.php?area $=060701$. Acesso em 07 ago. 2014.

Nascimento, T. G., Torres, C. V., \& Pimentel, C. E. (2011). Evidências de validade e precisão da escala de atitudes frente à polícia. Revista Brasileira de Segurança Pública, 5(9), 42-56.

Olson, M. A., \& Fazio, R. H. (2009). Implicit and explicit measures of attitudes: The perspective of the MODE model. In R. E. Petty, R. H. Fazio, \& P. Brinõl (Eds.), Attitudes: Insights from the new implicit measures (pp. 19-63). New York, NY: Psychology Press.

Pasquali, L. (2003). Psicometria: Teoria dos testes na psicologia e educação. Petrópolis, RJ: Vozes.

Peres, M. F. T. (2004). Violência por armas de fogo no Brasil: Relatório nacional. São Paulo: Núcleo de Estudos da Violência da Universidade de São Paulo.

Peres, M. F. T., \& Santos, P. C. (2005). Mortalidade por homicídios no Brasil na década de 90: O papel das armas de fogo. Revista de Saúde Pública, 39(1), 58-66. doi: 10.1590/S0034.

Peterson, R. A, \& Kim, Y. (2013). On the Relationship Between Coefficient Alpha and Composite Reliability. Journal of Applied Psychology. 98(1), 194-198. doi:10.1037/a0030767.

Pimentel, C. E. (2004). Valores humanos, preferência musical, identificação grupal e comportamentos anti-sociais. (Unpublished master's thesis), Universidade Federal da Paraíba.

Pimentel, C. E., Torres, C.V. \& Gunther, H. (2011). Estratégias de mensuração de atitudes em psicologia social. In C. V. Torres \& E. R. Neiva (Eds.), Psicologia social: Principais temas e vertentes (pp. 196-210). Porto Alegre: ArtMed.

Rodrigues, A., Assmar, E. M. L., \& Jablonski, B. (2012). Psicologia social. RJ, Rio de Janeiro: Editora Vozes.

Santos, W. S. (2008). Explicando comportamentos socialmente desviantes: Uma análise do compromisso convencional e afiliação social (Unpublished doctor dissertation), Universidade Federal da Paraíba / Universidade Federal do Rio Grande do Norte.

Sanches, S., Duarte, S. J. H., \& Pontes, E. R. J. C. (2009). Caracterização das vítimas de ferimentos por arma de fogo, atendidas pelo Serviço de Atendimento Móvel de Urgência em Campo Grande-MS. Saúde e Sociedade, 18(1), 95-102. doi:10.1590/S0104. 
Shapiro, J. P., Dorman, R. L., Burkey, W. M., Welker, C. J., \& Clough, J. B. (1997). Development and factor analysis of youth attitudes toward guns and violence. Journal of Clinical Child Psychology, 26(3), 311-320.

Shapiro, J. P., Dorman, R. L., Welker, C. J., \& Clough, J. B. (1998). Youth attitudes toward guns and violence: Relations with sex, age, ethnic group, and firearm exposure. Journal of Clinical Child Psychology, 72(1), 98-108.
Smith, T.W. (1980). The 75\% solution: An analysis of the structure of attitudes on gun control, 1959-1977. The Journal of Criminal Law \& Criminology, 71(3), 300-316.

Tabachnick, B. G., \& Fidell, L. S. (2007). Using multivariate statistics. Needham Heights, MA: Allyn \& Bacon.

Zwick, W. R., \& Velicer, W. F. (1986). Comparison of five rules for determining the number of components to retain. Psychological Bulletin, 99(3), 432-442. 